\title{
Hybrid Adsorptive and Oxidative Removal of Natural Organic Matter Using Iron Oxide-Coated Pumice Particles
}

\author{
Sehnaz Sule Kaplan Bekaroglu, ${ }^{1}$ Nevzat Ozgu Yigit, ${ }^{1}$ \\ Bilgehan Ilker Harman, ${ }^{2}$ and Mehmet Kitis ${ }^{1}$ \\ ${ }^{1}$ Department of Environmental Engineering, Suleyman Demirel University, 32260 Isparta, Turkey \\ ${ }^{2}$ Technical Science Vocational School, Suleyman Demirel University, 32260 Isparta, Turkey \\ Correspondence should be addressed to Sehnaz Sule Kaplan Bekaroglu; sulebekaroglu@sdu.edu.tr
}

Received 9 February 2016; Accepted 17 March 2016

Academic Editor: Angeles Val Del Rio

Copyright (C) 2016 Sehnaz Sule Kaplan Bekaroglu et al. This is an open access article distributed under the Creative Commons Attribution License, which permits unrestricted use, distribution, and reproduction in any medium, provided the original work is properly cited.

\begin{abstract}
The aim of this work was to combine adsorptive and catalytic properties of iron oxide surfaces in a hybrid process using hydrogen peroxide and iron oxide-coated pumice particles to remove natural organic matter (NOM) in water. Experiments were conducted in batch, completely mixed reactors using various original and coated pumice particles. The results showed that both adsorption and catalytic oxidation mechanisms played role in the removal of NOM. The hybrid process was found to be effective in removing NOM from water having a wide range of specific UV absorbance values. Iron oxide surfaces preferentially adsorbed UV $280^{-}$absorbing NOM fractions. Furthermore, the strong oxidants produced from reactions among iron oxide surfaces and hydrogen peroxide also preferentially oxidized $\mathrm{UV}_{280}$-absorbing NOM fractions. Preloading of iron oxide surfaces with NOM slightly reduced the further NOM removal performance of the hybrid process. Overall, the results suggested that the tested hybrid process may be effective for removal of NOM and control disinfection by-product formation.
\end{abstract}

\section{Introduction}

Disinfection by-products (DBPs) form in drinking water as a result of reactions between oxidants/disinfectants such as chlorine and natural organic matter (NOM). One of the major challenges for drinking water treatment is to control carcinogenic and mutagenic disinfection by-products (DBPs) formation. Today, more stringent standards are being imposed in developed countries in an effort to minimize the impacts of DBPs in public health. In addition, future regulations are expected to focus more on individual instead of combined DBPs because recent toxicology studies indicate that individual DBP species may have different health effects. In order to comply with current and future regulations, water utilities have been exploring various strategies to minimize the DBP formation. Two approaches are commonly used to meet the DBP regulations: (1) removal of the DBP precursors (i.e., DOM: dissolved organic matter which is the dissolved fraction of NOM) before chlorine addition and (2) use of alternative disinfectants/oxidants (e.g., ozone, chloramines, chlorine dioxide, or UV light) instead of chlorine. The use of alternative disinfectants may not be always feasible to apply in existing treatment plants, and they may produce other unfavorable disinfection by-products (e.g., nitrosamines) and water quality problems (e.g., nitrification and/or elevated lead levels in some distribution systems). Since reducing the precursor levels will result in lower degree of overall DBP formation, precursor control is the most commonly used and preferred method for DBP control.

Several studies have shown that iron oxides adsorb humic materials and NOM from water [1-9]. Furthermore, the surfaces of various metal oxide particles including iron oxides catalyze the decomposition of oxidants (e.g., ozone and hydrogen peroxide) resulting in the formation of strong oxidants such as hydroxyl radicals [10-18]. As in Fenton reactions, the decomposition of peroxide through interactions with the surface sites of such catalysts results in the formation of strong oxidants including hydroxyl 
radicals, which are shown to effectively oxidize various synthetic organic chemicals and NOM [14]. The combination effectiveness of $\mathrm{H}_{2} \mathrm{O}_{2}$ and iron-coated pumice combined processes has been attributed to the generation of highly reactive and nonselective hydroxyl radicals [16]. Therefore, iron oxide particles when added to water along with oxidants may remove NOM and/or synthetic chemicals through both adsorption and catalytic oxidation mechanisms. Zelmanov and Semiat [19] displayed the photochemical and catalytic properties of iron-based nanoparticles for use in degradation of some organic pollutants in wastewaters in the presence of hydrogen peroxide. Iron oxide particles added to hybrid ultrafiltration processes always improve both NOM removal and membrane flux [20].

Iron oxide can be immobilized on various support materials such as sand, soil, and zeolite. Pumice was selected as granular support medium for iron oxide coating for the purposes of this study. Pumice has been used as adsorbent and photocatalysts in water treatment $[7,16,21]$. As an alternative to other support materials such as sand, the advantages of pumice particles are that they are highly porous and have higher surface areas, which immobilize more amounts of metal oxide catalysts, thus providing more reaction sites. In our previous work, we investigated adsorptive NOM removal from water using iron oxide-coated natural pumice particles and found that, for all pumice particle size fractions, the coating of natural pumice with iron oxide significantly increased NOM uptake on both an adsorbent mass and surface area basis [7]. Furthermore, in another previous study, iron oxide-coated pumice particles were found to be effective in catalyzing the decomposition of hydrogen peroxide and removal of NOM from humic acid solution and a raw drinking water source with low specific UV absorbance $\left(\mathrm{SUVA}_{254}\right)$ value [16]. Therefore, based on the findings of our previous studies, the objective of this work was to combine both the adsorptive and catalytic properties of iron oxide-coated pumice particles in a hybrid process. The main objective was to investigate the effectiveness of this hybrid process in the removal of NOM from water. In our previous work [7], we investigated the adsorptive NOM removal from water using iron oxide-coated natural pumice particles. In our other previous study [16], the oxidative removal of natural organic matter with using hydrogen peroxide and iron-coated pumice particles was evaluated in a natural water with relatively low specific UV absorbance (SUVA) value $\left(\mathrm{SUVA}_{254} \mathrm{~nm}: 1.9 \mathrm{~L} / \mathrm{mg}-\mathrm{m}\right)$. The main difference of this study from previous published work [16] was to investigate the effectiveness of iron oxide-coated pumice and volcanic slag particles in removing DBP precursors from a high$\mathrm{SUVA}_{254}$ water. Natural water with $\mathrm{SUVA}_{254}$ values less than 2.0 generally contains mainly hydrophilic and low molecular weight NOM moieties $[22,23]$. On the other hand, water with higher SUVA254 values (i.e., >4 L/mg-m) mainly contains humic materials of higher molecular weight and hydrophobic character. Such water after chlorination may exert higher concentrations of disinfection by-products [22, 23]. Iron oxides have been shown to exhibit higher adsorption capacity for larger molecular size hydrophobic NOM fractions and acidic NOM fractions rich in carboxyl/hydroxyl functional groups such as aromatic moieties in humic materials [16]. Thus, it was hypothesized that iron oxide-coated pumice and slag particles will be more effective for NOM removal and DBP control in high-SUVA water. Natural surface water with a high-SUVA value and high dissolved organic carbon (DOC) concentration was chosen for this purpose. Raw water sample was obtained from the influent of drinking water treatment plant in Myrtle Beach (MB), South Carolina (SC), USA. NOM in MB water was concentrated using a pilot-scale reverse osmosis (RO) membrane system, which allowed conducting all adsorption experiments at a constant initial dissolved organic carbon (DOC) concentration (4.1$4.2 \mathrm{mg} / \mathrm{L})$ and $\mathrm{SUVA}_{254}(4.8 \mathrm{~L} / \mathrm{mg}-\mathrm{m})$. Furthermore, iron oxide-coated pumice particles were preloaded with NOM prior to the application of the hybrid process to evaluate the impact of the preloading of iron oxide surfaces on process performance.

\section{Experimental and Methods}

Three different natural pumice sources in Turkey (Isparta, Kayseri, and Nevsehir) with varying physicochemical characteristics were used in this study. The following codes are used throughout this paper for these pumice sources: Isp: Isparta; Kay: Kayseri; Nev: Nevsehir. Two different particle size fractions ( $<63$ and $250-1000 \mu \mathrm{m})$ were obtained for each pumice source after grinding and sieving. Pumice samples were used as received and coated with iron oxides. The pumice fractions were coated with iron oxides using reagent grade $\mathrm{FeCl}_{3} \cdot 6 \mathrm{H}_{2} \mathrm{O}$, employing the method reported by Lai et al. [24] and Lai and Chen [6] with some modifications. Detailed information on the employed coating procedures can be found in our previous publication [7]. Control experiments demonstrated that iron oxide precipitates/colloids were effectively removed from cleaning solutions during coating and that catalytic NOM oxidation was due to iron oxide-coated pumice particles not colloidal iron in the solution. Each uncoated (original) and coated pumice fraction was characterized by measuring specific surface area, point of zero charge $\left(\mathrm{pH}_{\mathrm{PZC}}\right)$, iron content, total surface acidic and basic groups, and scanning electron microscope, energy dispersive X-ray spectrometer (SEM-EDX) and X-ray fluorescence (XRF) analysis. Iron contents of the coated pumices were measured according to acid digestion analysis and with further AA spectrometry measurements. The detailed physicochemical characteristics of all pumice particles were presented elsewhere $[7,16]$. NOM used in this study was collected from the influent of Myrtle Beach (MB) drinking water treatment plant in South Carolina using a reverse osmosis (RO) membrane system to represent a raw water source with high DOC and SUVA values. The RO concentrate was diluted by distilled and deionized water (DDW) to obtain a constant initial DOC concentration $(4.1-4.2 \mathrm{mg} / \mathrm{L})$ for all experiments.

All hybrid process experiments were conducted in completely mixed batch reactors (CMBRs). First, kinetic experiments in CMBRs were performed with constant pumice $(3000 \mathrm{mg} / \mathrm{L})$ and peroxide $(300 \mathrm{mg} / \mathrm{L})$ dosages at periods of $1,2,4,8,12,24,36$, and $48 \mathrm{~h}$. The results indicated 
that no more statistically significant NOM removal occurred after $24 \mathrm{~h}$ of reaction with peroxide and iron oxide-coated pumice. Therefore, $24 \mathrm{~h}$ reaction period was employed for all remaining batch experiments. After kinetics experiments, determining the extent of NOM removal by adsorption and peroxide oxidation only, pumice and peroxide were also dosed alone. Then catalytic oxidation experiments were performed at various uncoated/coated pumice $(0-3000 \mathrm{mg} / \mathrm{L})$ and hydrogen peroxide $(0-1000 \mathrm{mg} / \mathrm{L})$ dosages. Three different natural pumice sources in Turkey (Isparta, Kayseri, and Nevsehir) with two different particle size fractions $(<63$ and $250-1000 \mu \mathrm{m}$ ) were tested to determine the effect of pumice source and particle size on NOM removals. The four different iron-coated pumice dosages $(30,100,1000$, and $3000 \mathrm{mg} / \mathrm{L})$ were examined to determine effects of catalyst dosages on decomposition of hydrogen peroxide. Three different NOM sources were used in the experiments: a surface water (Alibeykoy reservoir) supplying some portion of City of Istanbul's drinking water demand; a surface water obtained from the influent of drinking water treatment plant in Myrtle Beach (MB), South Carolina (SC), USA; and humic acid (HA) isolate purchased from Acros Organics. These NOM sources were selected since they represented a low- (Alibeykoy) and high-SUVA (MB and HA) water, enabling the evaluation of various pumices in removing NOM with a wide range of chemical characteristics. All tests were conducted in parallel CMBRs. Statistical analysis of the data was performed based on $t$-statistics and 95\% confidence intervals were calculated from parallel tests and triplicate measurements. CMBRs used were $255 \mathrm{~mL}$ glass amber bottles with PTFE screw-caps (solution volume: $200 \mathrm{~mL}$ ). All experiments were conducted at constant temperature of $20 \pm 1^{\circ} \mathrm{C}$. After dosing coated pumice and/or peroxide, CMBRs containing $\mathrm{MB}$ water were kept well mixed (150 rpm) in oxic conditions in a temperature-controlled orbital shaker. CMBRs were covered with aluminum foil to prevent the introduction of light. After employing the hybrid process, the bottles were opened and samples were taken to measure residual peroxide and determine the amount of sodium sulfite $\left(\mathrm{Na}_{2} \mathrm{SO}_{3}\right)$ solution required to quench the residual peroxide and stop the reactions. Solutions were filtered $(0.45 \mu \mathrm{m}$ membrane filter (polyethersulfone)) to remove pumice particles and analyzed for $\mathrm{pH}, \mathrm{UV}$ absorbance, and DOC concentration to quantify NOM removal. Filter papers were prewashed with $1 \mathrm{~L}$ of DDW to prevent potential leaching of materials from the filter matrix. Changes in DOC concentrations in control bottles (without peroxide and pumice dosing) were not statistically significant based on 95\% confidence intervals, indicating the stability of NOM during mixing. Kinetic experiments in CMBRs were performed with constant pumice $(3000 \mathrm{mg} / \mathrm{L})$ and peroxide $(300 \mathrm{mg} / \mathrm{L})$ dosages at periods of $1,2,4,8,12,24$, 36 , and $48 \mathrm{~h}$. The results indicated that no more statistically significant NOM removal occurred after $24 \mathrm{~h}$ of reaction with peroxide and iron oxide-coated pumice. Therefore, $24 \mathrm{~h}$ reaction period was employed for all remaining batch experiments.

Preloading experiments using $\mathrm{MB}$ water were conducted to evaluate the impacts of NOM preloading on further adsorptive and catalytic properties of iron oxide surfaces.
The smaller particle size fraction $(<63 \mu \mathrm{m})$ of coated Isp pumice was used for the preloading experiments. Three different $\mathrm{MB}$ raw water samples with different initial DOC concentrations $(1,5$, and $10 \mathrm{mg} / \mathrm{L})$ were prepared by diluting the $\mathrm{RO}$ concentrate of $\mathrm{MB}$ water using DDW. The preloading experiments were conducted in CMBRs. After dosing coated pumice dose of $2000 \mathrm{mg} / \mathrm{L}$ to each CMBR having different initial DOC concentration, CMBRs were mixed (150 rpm) in oxic conditions in a temperature-controlled orbital shaker for one week. Preliminary tests indicated that one week of mixing was highly sufficient to reach adsorption equilibrium on iron oxide surfaces. At the end of preloading experiments, water samples were taken and filtered prior to $\mathrm{UV}_{254}$ absorbance and DOC measurements. All pumice particles remaining on filter papers were collected and dried at $80^{\circ} \mathrm{C}$ in an oven until the moisture content was removed and constant weight was achieved. Using these preloaded coated pumice particles, hybrid process experiments were then conducted and the results were compared with those of nonpreloaded coated particles while other experimental variables were constant.

All chemicals used were of either analytical or reagent grade. DDW was used for stock solution preparations and dilutions. DOC concentrations were measured using a highsensitivity TOC analyzer (TOC-VCPH, Shimadzu) employing high-temperature combustion. A UV-visible spectrophotometer (UV-1601, Shimadzu) was used to measure the UV absorbances (in triplicate) in water samples. Hydrogen peroxide concentration was measured with a titrimetric test kit (HYP-1, Hach-Lange).

\section{Results and Discussion}

The SEM images of original and iron oxide-coated Isp pumice particles are shown in Figures 1(a) and 1(b), respectively. The porous structure of original pumice particles and partial coverage/filling of these pores by the coating can be clearly seen in these images. Similar to our SEM results, the study shows that original pumice surfaces were apparently occupied by iron oxides, which were formed during the coating process [25]. Various physicochemical characteristics of all pumice particles were presented in detail in our previous publications $[7,16]$.

Initially, experiments were conducted by dosing hydrogen peroxide alone to determine the degree of NOM removal in $\mathrm{MB}$ raw water by peroxide oxidation only. The results showed that NOM removal by peroxide oxidation was minimal and less than a $6 \%$ reduction in $\mathrm{UV}_{254}$ absorbance was achieved with peroxide dosages up to $1000 \mathrm{mg} / \mathrm{L}$ (Figure 2(a)). Similar results were also found for DOC (Figure $2(\mathrm{~b})$ ). These findings were expected since peroxide is known to be generally ineffective in oxidizing refractory synthetic chemicals or NOM. For all the tested original (uncoated) pumice sources and particle size fractions, the adsorptive removal of NOM in MB water without peroxide was also minor. The maximum $\mathrm{UV}_{254}$ absorbance and DOC reductions achieved via uncoated particles were $10 \%$ and $5 \%$, respectively, even at maximum dosages. On the other hand, the coating of pumice particles with iron oxide significantly enhanced adsorptive NOM removal. When a dose 


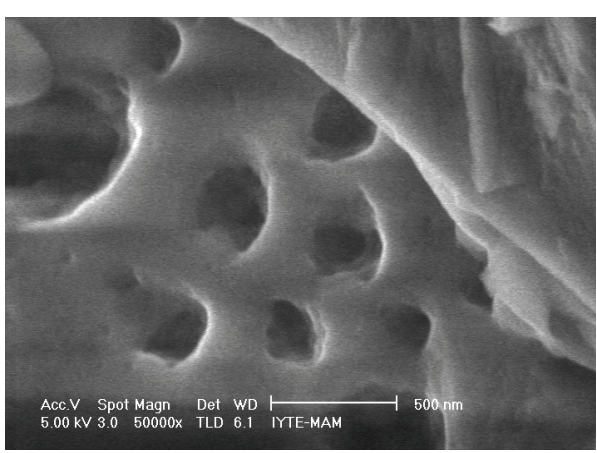

(a)

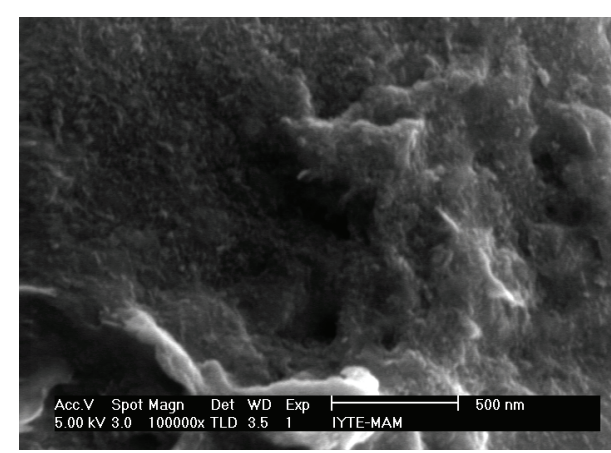

(b)

FIGURE 1: SEM images of uncoated/original (a) and iron oxide-coated (b) Isp pumice particles (size fraction: $<63 \mu \mathrm{m}$ ).

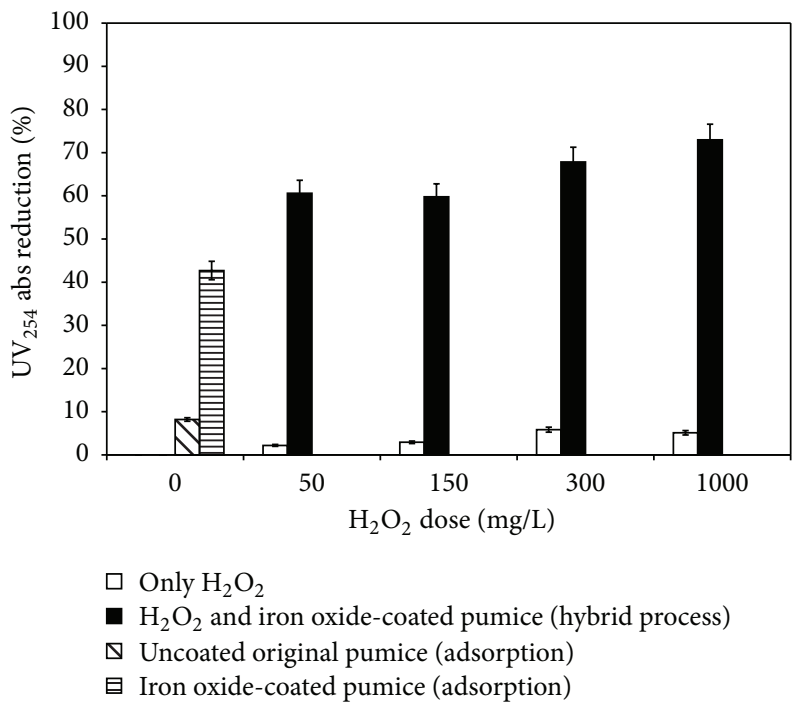

(a)

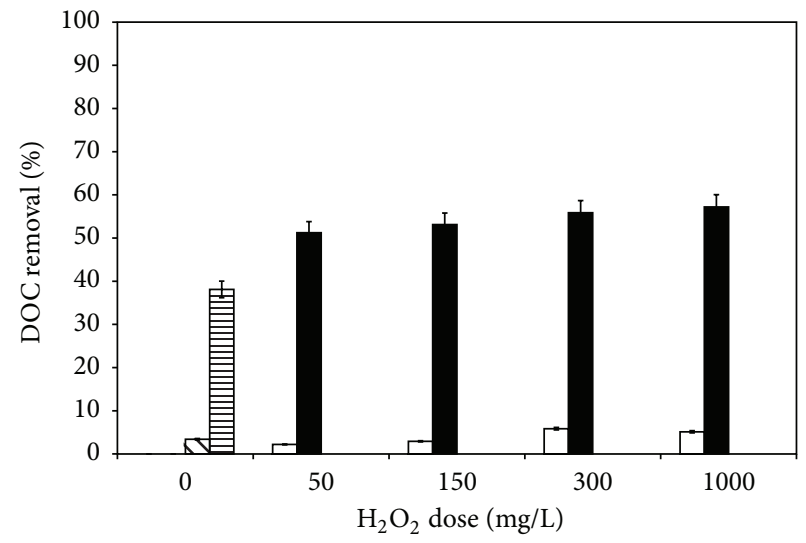

$\square$ Only $\mathrm{H}_{2} \mathrm{O}_{2}$

- $\mathrm{H}_{2} \mathrm{O}_{2}$ and iron-coated pumice (hybrid process)

$\checkmark$ Uncoated original pumice (adsorption)

目 Iron-coated pumice (adsorption)

(b)

FIGURE 2: NOM removals in MB water by the original or iron oxide-coated pumice particles (adsorption only), hydrogen peroxide alone, and the hybrid process (iron oxide-coated pumice and peroxide). UV reductions (a) and DOC removal (b). $<63 \mu \mathrm{m}$ Isp pumice, pumice dose: $3000 \mathrm{mg} / \mathrm{L}$, reaction period: $24 \mathrm{~h}$, and initial DOC: $4.1 \mathrm{mg} / \mathrm{L}$. Error bars indicate the $95 \%$ confidence intervals.

of $3000 \mathrm{mg} / \mathrm{L}$ coated pumice was used, $\mathrm{UV}_{254}$ absorbance and DOC removals as high as 43 and $36 \%$ were obtained, respectively, in all tested pumice types and size fractions. These results overall suggest that iron oxide-coated pumice particles may be effective in the adsorptive removal of NOM in water with high-SUVA ${ }_{254}$ values $(4.84 \mathrm{~L} / \mathrm{mg}-\mathrm{m}$ for the tested $\mathrm{MB}$ water). In our previous work [7], iron oxidecoated pumice or volcanic slag particles were also found to be effective adsorbents in removal of NOM in water samples with lower $\mathrm{SUVA}_{254}$ values $(<2.0 \mathrm{~L} / \mathrm{mg}-\mathrm{m})$. While SUVA 254 values less than $2.0(\mathrm{~L} / \mathrm{mg}-\mathrm{m})$ generally indicate that NOM is mainly of hydrophilic character with lower molecular weight fractions (i.e., nonhumic materials), $\mathrm{SUVA}_{254}$ values higher than $4.0(\mathrm{~L} / \mathrm{mg}-\mathrm{m})$ indicate water with dominantly humic materials with higher molecular weight and higher degree of aromaticity $[23,24]$. Thus, iron oxide-coated pumice particles appear to be effective adsorbents for a wide range of raw water sources having different NOM moieties.
When hydrogen peroxide and iron oxide-coated pumice particles were dosed together, both $\mathrm{UV}_{254}$ absorbance and DOC removal further increased. A $73 \%$ reduction in $\mathrm{UV}_{254}$ absorbance was achieved with iron oxide-coated Isp pumice $(<63 \mu \mathrm{m})$ and peroxide doses of $3000 \mathrm{mg} / \mathrm{L}$ and $1000 \mathrm{mg} / \mathrm{L}$, respectively (Figure 2(a)). The DOC removal was $57 \%$ at these doses (Figure 2(b)). Increased NOM removal was detected for all coated pumice types and size fractions when dosed with peroxide. Furthermore, as the peroxide dosages were increased at a constant coated pumice dose, both $\mathrm{UV}_{254}$ absorbance and DOC reductions also increased. These results prove that in addition to the adsorbent properties iron oxide surfaces also catalyze the decomposition of hydrogen peroxide resulting in the formation of strong oxidants, probably hydroxyl radicals. Thus, it is apparent that both adsorption and surface catalytic oxidation mechanisms play a role in the removal of NOM by this hybrid process. Control experiments prove that iron oxide species bound on pumice surfaces have 


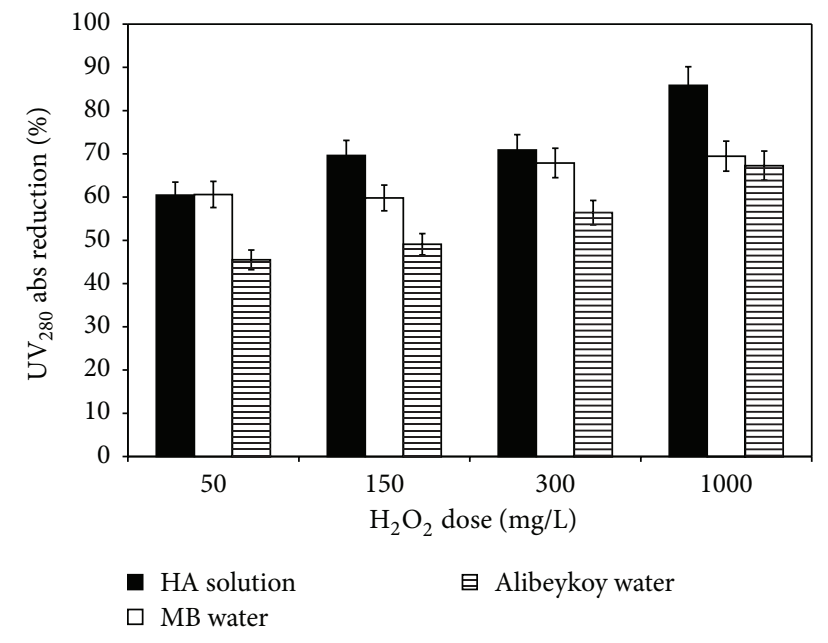

FIGURE 3: NOM removal performances of the hybrid process in three different water sources. $<63 \mu \mathrm{m}$ iron oxide-coated Isp pumice, pumice dose: $3000 \mathrm{mg} / \mathrm{L}$, HA: humic acid solution. Error bars indicate the $95 \%$ confidence intervals.

effective coating and stability. Total iron release to solution was always less than $0.15 \mathrm{mg} / \mathrm{L}$ at $\mathrm{pH}$ values $5.5-8.5$ even at a maximum dose of coated pumice $(3000 \mathrm{mg} / \mathrm{L})$ after $24 \mathrm{~h}$ of mixing (peroxide: $150 \mathrm{mg} / \mathrm{L}$ ). This finding indicated two important points: (1) potential iron release to water in this hybrid process is not a concern at neutral $\mathrm{pH}$ values of typical natural water; (2) strong oxidants are produced by the coated iron oxides on pumice surfaces, not by colloidal or soluble iron species in water.

Figure 3 shows the $\mathrm{UV}_{280}$ absorbance reductions achieved by this hybrid process in different water sources. Some of the results for Alibeykoy raw water (Istanbul, Turkey) and humic acid (HA) solution were presented in our previous publication [16]. Since sodium azide was added to Alibeykoy water and HA solution to prevent microbial NOM degradation during their long storage, $\mathrm{UV}_{280}$ absorbance data were given in Figure 3. This is because while sodium azide absorbs UV light at $254 \mathrm{~nm}$ wavelength it does not absorb UV at $280 \mathrm{~nm}$. Therefore, $\mathrm{UV}_{280}$ absorbance measurements were used to compare the NOM removal performances in these water samples. It was found that the degree of NOM removal by the hybrid process generally increased along with increasing SUVA $_{280}$ values in the tested water samples (Alibeykoy water: 1.41, $\mathrm{MB}$ water: 3.64, and $\mathrm{HA}$ solution: $5.11 \mathrm{~L} / \mathrm{mg}-$ $\mathrm{m}$ ) (Figure 3). $\mathrm{UV}_{280}$ absorbance reductions achieved in the Alibeykoy water, MB water, and HA solution were 49, 60, and $70 \%$, respectively, employing a dose of $150 \mathrm{mg} / \mathrm{L}$ peroxide and $3000 \mathrm{mg} / \mathrm{L}$ iron oxide-coated pumice. At the same doses, the corresponding DOC removals were 22, 53, and 61\%. Higher DOC and $\mathrm{UV}_{280}$ absorbance reductions were achieved with increasing peroxide dosages from 150 to $1000 \mathrm{mg} / \mathrm{L}$. For example, when doses of $1000 \mathrm{mg} / \mathrm{L}$ peroxide and $3000 \mathrm{mg} / \mathrm{L}$ coated pumice were used, the DOC removals achieved in the Alibeykoy water, $\mathrm{MB}$ water, and $\mathrm{HA}$ solution have been 35,57 , and $73 \%$, respectively. Alver et al. [25] studied the catalytic activity of the iron-coated pumice particles used as heterogeneous catalysts in the oxidation of humic acid solution. Consistent with our results, they reported that DOC reduction reached $74 \%$ at the maximum iron-coated pumice dosage $(5000 \mathrm{mg} / \mathrm{L})$ and the maximum $\mathrm{H}_{2} \mathrm{O}_{2}$ dosage (200 mg/L) whereas for adsorption alone this was $11 \%$. These findings indicate that (1) the hybrid process is effective for a wide range of natural water sources having SUVA $_{280}$ values from 1.41 to $5.11 \mathrm{~L} / \mathrm{mg}-\mathrm{m}$ and (2) a higher degree of NOM removal can be achieved in water sources with dominantly humic materials rich in aromatic structures. All experiments were conducted at the $\mathrm{pH}$ of original solution (8.0, 7.1, and 6.8 for Alibeykoy, M. Beach, and humic acid, resp.) without any buffer and constant temperature of $20 \pm 1^{\circ} \mathrm{C}$. Final $\mathrm{pH}$ values after experiments were between 7.8-8.0, 6.2-7.6, and 6.47.2 for Alibeykoy water, M. Beach, and humic acid solution, respectively.

It was observed that $\mathrm{SUVA}_{280}$ values decreased following the application of hybrid process with increasing peroxide and coated pumice doses in all the tested water samples. This trend suggests that (1) iron oxide surfaces preferentially adsorb $\mathrm{UV}_{280}$-absorbing NOM fractions such as aromatic moieties in humic materials, consistent with previous studies $[3,5,7]$, and (2) the strong oxidants produced as a result of surface reactions of iron oxides and hydrogen peroxide also preferentially oxidize $\mathrm{UV}_{280}$-absorbing NOM fractions in solutions. It was difficult to exactly determine which mechanism contributed more to the preferential removal of $\mathrm{UV}_{280}$-absorbing NOM fractions. This is due to the observed synergistic effects and the possibility that adsorbed $\mathrm{UV}_{280^{-}}$ absorbing NOM fractions on iron oxide surfaces may have oxidized as a result of surface reactions. Nevertheless, the hybrid process preferentially removed the $\mathrm{UV}_{280}$-absorbing NOM fractions by the above-mentioned mechanisms, which is very important since the major disinfection by-product (DBP) precursors during chlorination are believed to be UVabsorbing NOM fractions. Alver et al. [25] reported that the removal of trihalomethane formation potential (THMFP) was $65 \%$ for $100 \mathrm{mg} / \mathrm{L}_{2} \mathrm{O}_{2}$ and $2000 \mathrm{mg} / \mathrm{L}$ iron-coated pumice dosage for HA solutions. Thus, this property of the hybrid process may be useful in terms of controlling DBP formations in drinking water treatment.

Many researchers $[26,27]$ have reported the catalytic oxidation of $\mathrm{HA}$ solutions by iron-coated pumice $/ \mathrm{H}_{2} \mathrm{O}_{2}$ process that the initial $\mathrm{H}_{2} \mathrm{O}_{2}$ concentration played a very important role for the generation of hydroxyl radicals to oxidation of NOM. NOM removal in MB water increased as coated pumice and peroxide doses were increased. At constant pumice dose, UV254 absorbance reductions increased when peroxide doses were increased. Similarly, at constant peroxide doses, $\mathrm{UV}_{254}$ absorbance reductions increased when pumice dose was increased. Furthermore, the ratio of $\mathrm{H}_{2} \mathrm{O}_{2}$ /coated pumice dosage $(\mathrm{mg} / \mathrm{mg}$ ) also impacted NOM removal; increasing this ratio also increased NOM removal (Figure 4). The range of $\mathrm{H}_{2} \mathrm{O}_{2}$ /coated pumice dose ratio tested in $\mathrm{MB}$ water was $0.02-33$. About $73 \% \mathrm{UV}_{254}$ absorbance and $57 \%$ DOC reductions were achieved when this ratio was 0.33 (1000 mg/L peroxide and $3000 \mathrm{mg} / \mathrm{L}$ coated pumice dose). This ratio may be optimized based on target DOC removal and cost consideration in a specific application. For each 


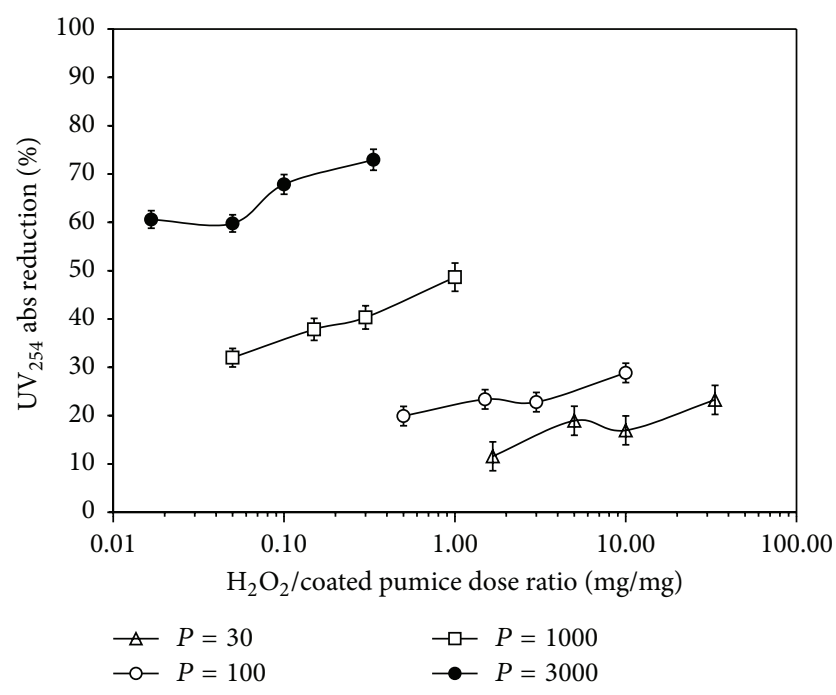

Figure 4: The effects of $\mathrm{H}_{2} \mathrm{O}_{2}$ /iron oxide-coated pumice dose ratio $(\mathrm{mg} / \mathrm{mg})$ on NOM removals in MB water. $<63 \mu \mathrm{m}$ iron oxide-coated Isp pumice: pumice dosages in the legend are in $\mathrm{mg} / \mathrm{L}$. Error bars indicate the $95 \%$ confidence intervals.

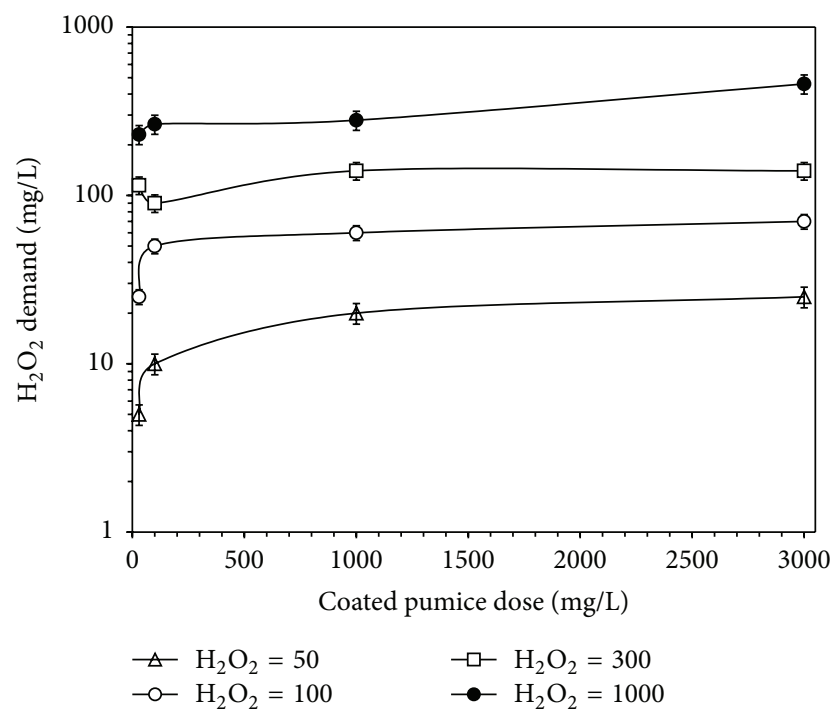

FIGURE 5: The effects of iron oxide-coated pumice and hydrogen peroxide dosages on hydrogen peroxide demands in $\mathrm{MB}$ water. $<63 \mu \mathrm{m}$ iron oxide-coated Isp pumice: peroxide dosages in the legend are in $\mathrm{mg} / \mathrm{L}$. Error bars indicate the $95 \%$ confidence intervals.

peroxide dose, peroxide consumption generally increased with increasing coated pumice dose (Figure 5). In addition, positive correlations were found between peroxide consumption and $\mathrm{UV}_{254}$ absorbance or DOC reductions at each pumice dosage level (data not shown). These two findings further proved that iron oxide surfaces are responsible for the catalytic decomposition of peroxide and further production of strong oxidants and that the oxidative NOM removals are directly linked to the produced oxidants. If NOM removals had not increased with increasing peroxide consumption, then this would indicate that NOM removals were only through adsorption. Another evidence supporting this is

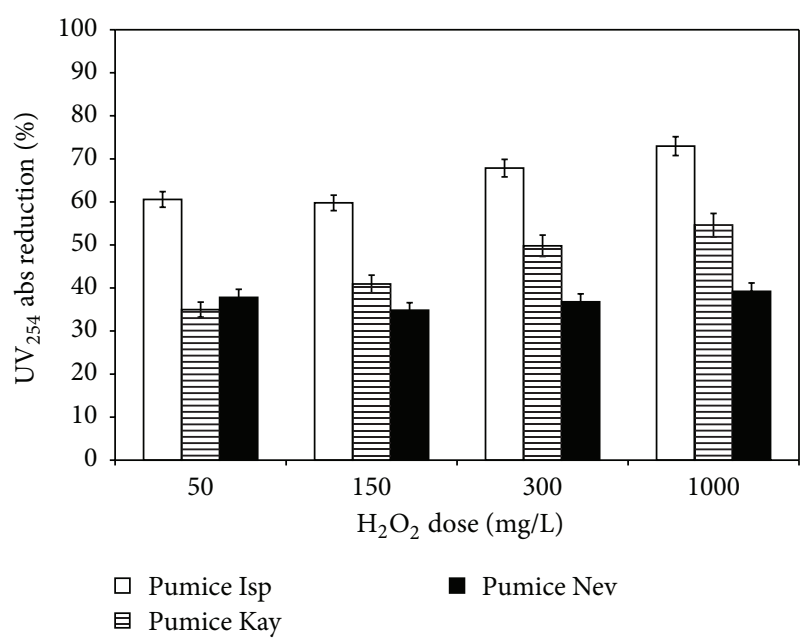

FIgURE 6: The effects of the pumice source on NOM removal performances of the hybrid process. $<63 \mu \mathrm{m}$ iron oxide-coated pumice, pumice dose: $3000 \mathrm{mg} / \mathrm{L}$. Error bars indicate the $95 \%$ confidence intervals.

that no correlation among peroxide consumption and NOM removal is observed when only peroxide is dosed (no coated pumice). Overall, the results show that NOM removal by the iron oxide-coated pumice and peroxide is the result of a hybrid process of adsorption and catalytic oxidation.

The effects of pumice source on NOM removals are shown in Figure 6. For both particle size fractions tested, iron oxide-coated Isp pumice provided the highest extent of $\mathrm{UV}_{254}$ absorbance and DOC removals among all pumice sources. This result is mainly because (1) the most effective coating as measured by iron contents was achieved in Isp pumice particles and (2) Isp pumice particles had generally higher specific surface areas. A strong linear correlation $\left(R^{2}=\right.$ 0.99) was found between iron contents of coated pumice particles and $\mathrm{UV}_{254}$ absorbance reductions. The iron contents of $<63 \mu \mathrm{m}$ coated Isp, Kay, and Nev pumices were 16.2, 13.4, and $11.1 \mathrm{mg} \mathrm{Fe} / \mathrm{g}$, respectively. The specific surface areas of $<63 \mu \mathrm{m}$ coated Isp, Kay, and Nev pumices were 12.9, 12.4, and $10.1 \mathrm{~m}^{2} / \mathrm{g}$, respectively. As shown in Figure $6, \mathrm{UV}_{254}$ absorbance reductions achieved by different pumice sources were generally in the following order: Isp $>$ Kay $>$ Nev pumice particles. This trend is consistent with the iron content and specific surface area data presented above. Overall, these data indicate that the mass amount of iron oxides coated on surfaces and the specific pumice surface area are important factors for NOM removal performance of the hybrid process. Apparently, as the values of both factors increase more reaction sites become available for producing strong oxidants. In addition, the adsorptive removal of NOM also increases due to more available sorption sites.

Iron oxide-coated pumice particles were preloaded with NOM prior to the hybrid process to evaluate the impact of preloading of iron oxide surfaces on process performance. Iron oxide surfaces may become filled/saturated as a result of NOM adsorption during the hybrid process, if the adsorbed NOM moieties on iron oxide surfaces are not continuously 
oxidized and removed by the produced strong oxidants. Thus, the catalytic and oxidant production properties of iron oxide surfaces may diminish by time due to NOM adsorption. In an effort to test this hypothesis, hybrid process experiments in $\mathrm{MB}$ water were conducted using preloaded or nonpreloaded coated pumice particles employing $1000 \mathrm{mg} / \mathrm{L}$ pumice and peroxide dosages. The results showed that preloading of iron oxide surfaces reduced the NOM removal performance of the hybrid process only about $7-14 \%$ (as measured by DOC and $\mathrm{UV}_{254}$ absorbance). Such reductions in NOM removal performances further increased as the initial DOC concentrations were increased during preloading. The reductions in DOC removals with respect to those achieved by nonpreloaded particles were 7,12 , and $14 \%$ for the initial DOC concentrations of 1,5 , and $10 \mathrm{mg} / \mathrm{L}$ during preloading, respectively. In other words, as the iron oxide surfaces were preloaded with higher masses of DOC, DOC removal performance of the hybrid process further decreased. Thus, the results indicated that iron oxide sites were partially covered with the adsorbed NOM, which reduced the catalytic and oxidant production properties of iron oxide surfaces. However, the negative impact of preloading can be considered minimal since only $14 \%$ reduction in DOC removal performance was found even at the highest degree of preloading. Further work will be conducted to determine regeneration protocols and efficiencies for the coated pumice particles.

\section{Concluding Remarks}

Both the adsorptive and catalytic properties of iron oxide surfaces were combined in a hybrid process by using hydrogen peroxide and iron oxide-coated pumice particles to remove NOM from water. The results show that both adsorption and catalytic oxidation mechanisms play a role in the removal of NOM. Iron oxide surfaces on pumice particles effectively catalyzed the decomposition of hydrogen peroxide resulting in the formation of strong oxidants. Release of iron to water during the hybrid process was negligible at $\mathrm{pH}$ values 5.58.5 even at the maximum coated pumice and peroxide doses. The hybrid process was effective in removing NOM from water sources with a wide range of $\mathrm{SUVA}_{280}$ values ranging from 1.41 to $5.11 \mathrm{~L} / \mathrm{mg}-\mathrm{m}$. It was found that iron oxide surfaces preferentially adsorbed $\mathrm{UV}_{280}$-absorbing NOM fractions. In addition, strong oxidants produced as a result of surface reactions between iron oxides and hydrogen peroxide also preferentially oxidized $\mathrm{UV}_{280}$-absorbing NOM fractions in tested water samples. This property of the hybrid process may be useful in terms of controlling DBP formations in drinking water treatment.

The area of iron oxide-coated surfaces and specific pumice surface area also proved to be important factors since more adsorbing- and oxidant-producing-sites became available as the values for these factors increased. Preloading of iron oxide surfaces with NOM slightly reduced further NOM removal performance of the hybrid process, indicating that iron oxide sites were partially covered with the adsorbed NOM, which reduced the catalytic and oxidant production properties of iron oxide surfaces. However, the negative impact of preloading was minimal; only $14 \%$ less DOC removal was detected even at maximum degree of preloading. In the next phase of the project, long-term experiments for the hybrid process will be conducted in both continuous flow fixed-bed and completely mixed batch reactor configurations. Thus, the long-term performance of the process and the potential negative impacts of the irreversible saturation of iron oxide surfaces on the catalytic properties will be investigated in detail. Furthermore, since the iron oxides on pumice surfaces behaving as both a catalyst and an adsorbent may need regeneration due to NOM adsorption, further tests will be conducted to determine regeneration protocols and efficiencies.

\section{Competing Interests}

The authors declare that they have no competing interests.

\section{Acknowledgments}

This work was supported by a Research Grant (Career Award Program, CAYDAG 104I122) from the Scientific and Technical Research Council of Turkey (TUBITAK). The authors thank Professor Tanju Karanfil and his research group at Clemson University, USA, for all of their contributions to the project.

\section{References}

[1] R. L. Parfitt, A. R. Fraser, and V. C. Farmer, "Adsorption on hydrous oxides. III. fulvic acid and humic acid on goethite, gibbsite and imogolite," Journal of Soil Science, vol. 28, no. 2, pp. 289-296, 1977.

[2] E. Tipping, "The adsorption of aquatic humic substances by iron oxides," Geochimica et Cosmochimica Acta, vol. 45, no. 2, pp. 191199, 1981.

[3] B. Gu, J. Schmitt, Z. Chen, L. Liang, and J. F. McCarthy, "Adsorption and desorption of natural organic matter on iron oxide: mechanisms and models," Environmental Science and Technology, vol. 28, no. 1, pp. 38-46, 1994.

[4] Y. Chang, C.-W. Li, and M. M. Benjamin, "Iron oxide-coated media for NOM sorption and particulate filtration," Journal of the American Water Works Association, vol. 89, no. 5, pp. 100$113,1997$.

[5] G. V. Korshin, M. M. Benjamin, and R. S. Sletten, "Adsorption of natural organic matter (NOM) on iron oxide: effects on NOM composition and formation of organo-halide compounds during chlorination," Water Research, vol. 31, no. 7, pp. $1643-$ 1650, 1997.

[6] C. H. Lai and C. Y. Chen, "Removal of metal ions and humic acid from water by iron-coated filter media," Chemosphere, vol. 44, no. 5, pp. 1177-1184, 2001.

[7] M. Kitis, S. S. Kaplan, E. Karakaya, N. O. Yigit, and G. Civelekoglu, "Adsorption of natural organic matter from waters by iron coated pumice," Chemosphere, vol. 66, no. 1, pp. 130-138, 2007.

[8] S. Kumpulainen, F. von der Kammer, and T. Hofmann, "Humic acid adsorption and surface charge effects on schwertmannite and goethite in acid sulphate waters," Water Research, vol. 42, no. 8-9, pp. 2051-2060, 2008 
[9] X. Shuai and G. Zinati, "Proton charge and adsorption of humic acid and phosphate on goethite," Soil Science Society of America Journal, vol. 73, no. 6, pp. 2013-2020, 2009.

[10] N. Al-Hayek and M. Doré, "en Oxidation of phenols in water by hydrogen peroxide on alumine supported iron," Water Research, vol. 24, no. 8, pp. 973-982, 1990.

[11] S.-S. Lin and M. D. Gurol, "Heterogeneous catalytic oxidation of organic compounds by hydrogen peroxide," Water Science and Technology, vol. 34, no. 9, pp. 57-64, 1996.

[12] S.-S. Lin and M. D. Gurol, "Catalytic decomposition of hydrogen peroxide on iron oxide: kinetics, mechanism, and implications," Environmental Science and Technology, vol. 32, no. 10, pp. 1417-1423, 1998.

[13] M. D. Gurol and S.-S. Lin, "Hydrogen peroxide/iron oxideinduced catalytic oxidation of organic compounds," Water Science and Technology: Water Supply, vol. 1, no. 4, pp. 131-138, 2001.

[14] E. Neyens and J. Baeyens, "A review of classic Fenton's peroxidation as an advanced oxidation technique," Journal of Hazardous Materials, vol. 98, no. 1-3, pp. 33-50, 2003.

[15] B. Kasprzyk-Hordern, U. Raczyk-Stanisławiak, J. Świetlik, and J. Nawrocki, "Catalytic ozonation of natural organic matter on alumina," Applied Catalysis B: Environmental, vol. 62, no. 3-4, pp. 345-358, 2006.

[16] M. Kitis and S. S. Kaplan, "Advanced oxidation of natural organic matter using hydrogen peroxide and iron-coated pumice particles," Chemosphere, vol. 68, no. 10, pp. 1846-1853, 2007.

[17] I. R. Guimarães, L. C. A. Oliveira, P. F. Queiroz et al., "Modified goethites as catalyst for oxidation of quinoline: evidence of heterogeneous Fenton process," Applied Catalysis A: General, vol. 347, no. 1, pp. 89-93, 2008.

[18] F. Qi, B. Xu, Z. Chen, J. Ma, D. Sun, and L. Zhang, "Influence of aluminum oxides surface properties on catalyzed ozonation of 2,4,6-trichloroanisole," Separation and Purification Technology, vol. 66, no. 2, pp. 405-410, 2009.

[19] G. Zelmanov and R. Semiat, "Phenol oxidation kinetics in water solution using iron(3)-oxide-based nano-catalysts," Water Research, vol. 42, no. 14, pp. 3848-3856, 2008.

[20] P. Yao, K.-H. Choo, and M.-H. Kim, "A hybridized photocatalysis-microfiltration system with iron oxide-coated membranes for the removal of natural organic matter in water treatment: effects of iron oxide layers and colloids," Water Research, vol. 43, no. 17, pp. 4238-4248, 2009.

[21] N. O. Yigit and S. Tozum, "Removal of selenium species from waters using various surface-modified natural particles and waste materials," Clean-Soil, Air, Water, vol. 40, no. 7, pp. 735745, 2012.

[22] M. Kitis, T. Karanfil, and J. E. Kilduff, "The reactivity of dissolved organic matter for disinfection by-product formation," Turkish Journal of Engineering and Environmental Sciences, vol. 28, no. 3, pp. 167-179, 2004.

[23] N. Ates, M. Kitis, and U. Yetis, "Formation of chlorination byproducts in waters with low SUVA-correlations with SUVA and differential UV spectroscopy," Water Research, vol. 41, no. 18, pp. 4139-4148, 2007.

[24] C. H. Lai, S. L. Lo, and H. L. Chiang, "Adsorption/desorption properties of copper ions on the surface of iron-coated sand using BET and EDAX analyses," Chemosphere, vol. 41, no. 8, pp. 1249-1255, 2000.
[25] A. Alver, M. Karaarslan, and A. K1lıç, "The catalytic activity of the iron-coated pumice particles used as heterogeneous catalysts in the oxidation of natural organic matter by $\mathrm{H}_{2} \mathrm{O}_{2}$," Environmental Technology, 2016.

[26] H. R. Sindelar, M. T. Brown, and T. H. Boyer, "Evaluating $\mathrm{UV} / \mathrm{H}_{2} \mathrm{O}_{2}, \mathrm{UV} /$ percarbonate, and $\mathrm{UV} /$ perborate for natural organic matter reduction from alternative water sources," Chemosphere, vol. 105, pp. 112-118, 2014.

[27] W. Yang, L. Dong, Z. Luo et al., "Application of ultrasound and quartz sand for the removal of disinfection byproducts from drinking water," Chemosphere, vol. 101, pp. 34-40, 2014. 

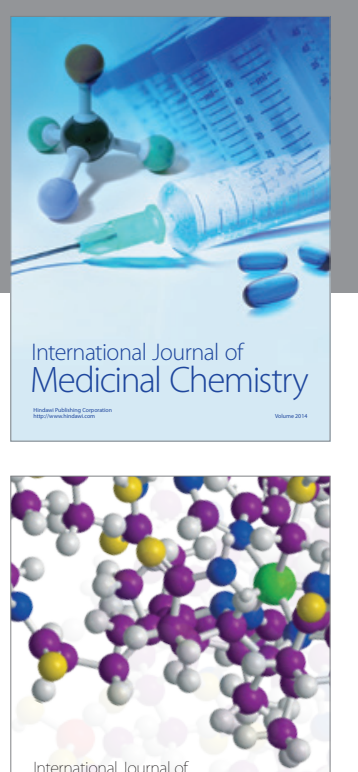

Carbohydrate Chemistry

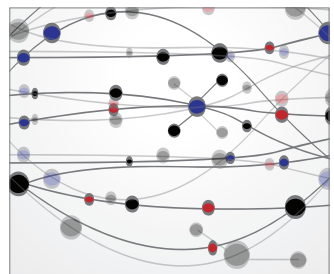

The Scientific World Journal
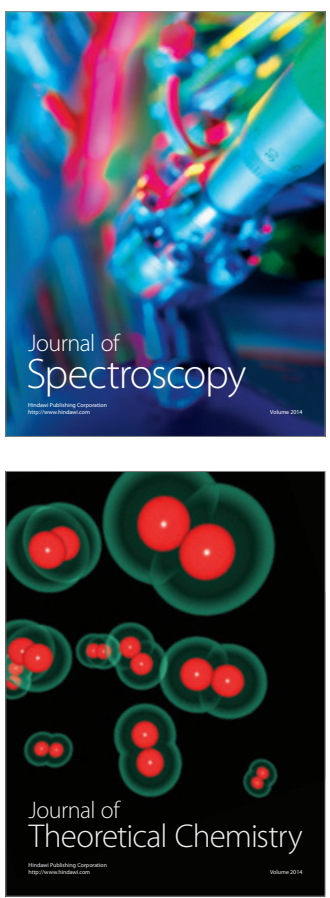
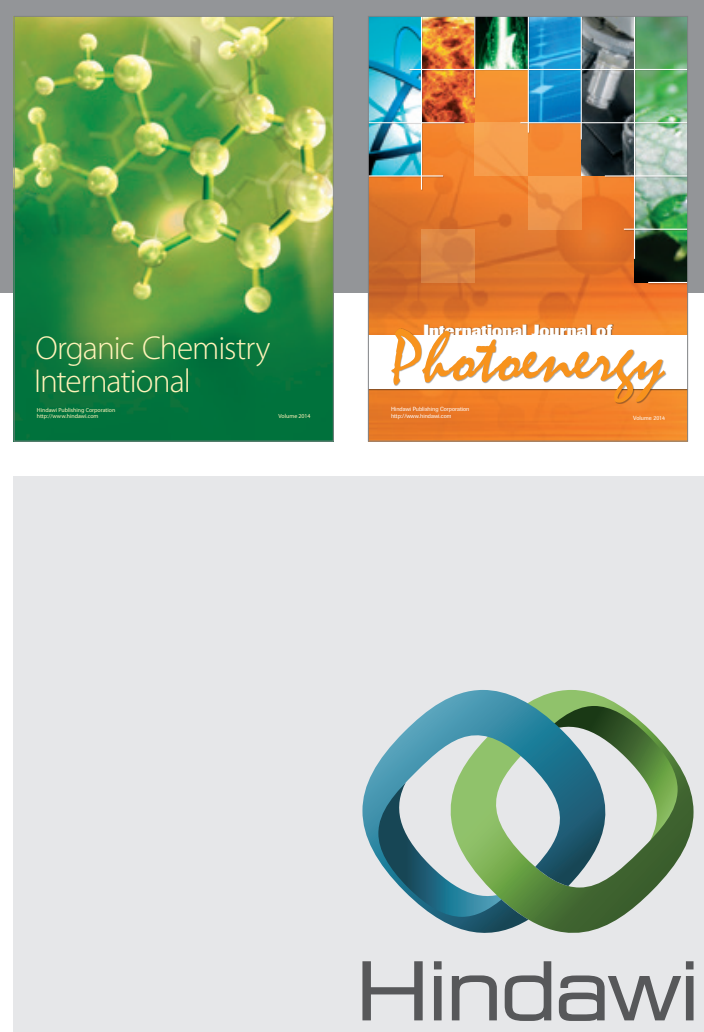

Submit your manuscripts at

http://www.hindawi.com

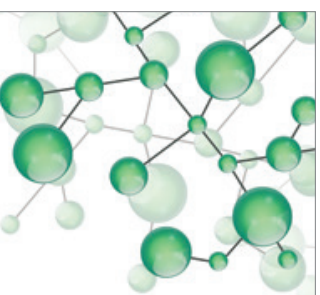

International Journal of

Inorganic Chemistry

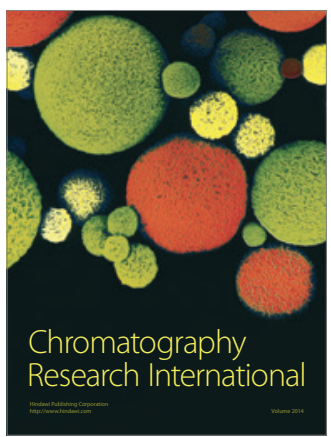

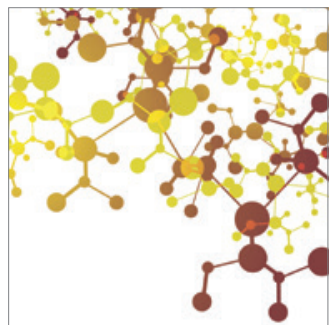

Applied Chemistry
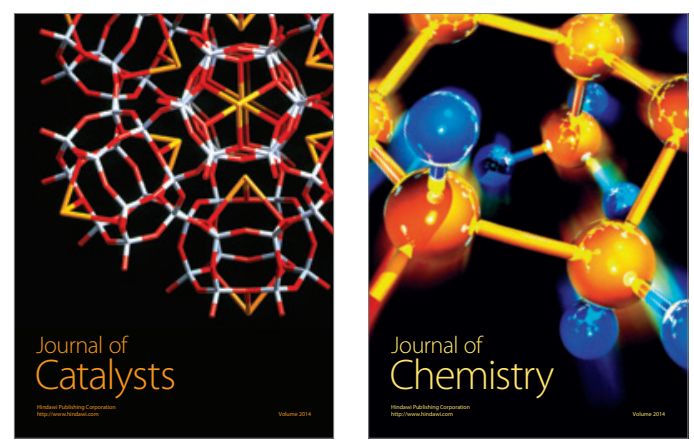
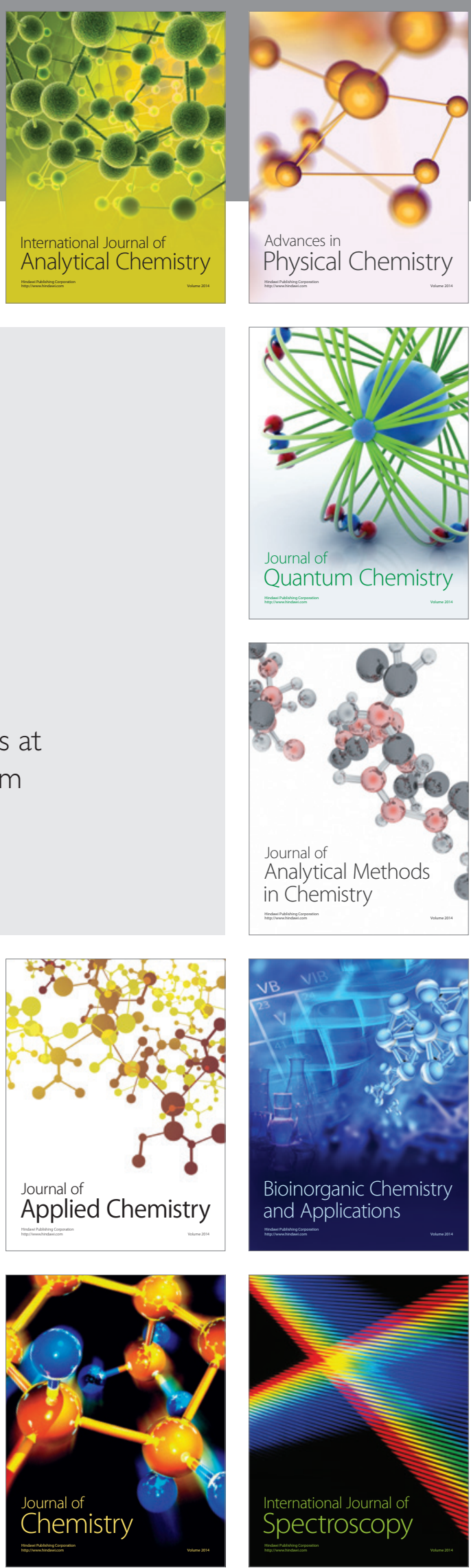